Review

\title{
Ribonucleoprotein Complexes That Control Circadian Clocks
}

\section{Dongni Wang, Xiaodi Liang, Xianyun Chen and Jinhu Guo *}

Key Laboratory of Gene Engineering of the Ministry of Education, State Key Laboratory of Biocontrol, School of Life Sciences, Sun Yat-sen University, Guangzhou 510275, China; E-Mails: wdn001@sina.cn (D.W.); sodylong@sina.com (X.L.); chenxianyun3000@sina.com (X.C.)

* Author to whom correspondence should be addressed; E-Mail: guojinhu@mail.sysu.edu.cn; Tel./Fax: +86-20-3933-2939.

Received: 28 January 2013; in revised form: 7 April 2013 / Accepted: 15 April 2013 /

Published: 25 April 2013

\begin{abstract}
Circadian clocks are internal molecular time-keeping mechanisms that enable organisms to adjust their physiology and behavior to the daily surroundings. Misalignment of circadian clocks leads to both physiological and health impairment. Post-transcriptional regulation and translational regulation of circadian clocks have been extensively investigated. In addition, accumulating evidence has shed new light on the involvement of ribonucleoprotein complexes (RNPs) in the post-transcriptional regulation of circadian clocks. Numerous RNA-binding proteins (RBPs) and RNPs have been implicated in the post-transcriptional modification of circadian clock proteins in different model organisms. Herein, we summarize the advances in the current knowledge on the role of RNP complexes in circadian clock regulation.
\end{abstract}

Keywords: ribonucleoprotein; circadian clock; transcription; translation; post-transcription

\section{Introduction}

Due to the daily self-rotation of the Earth, environmental factors (e.g., light, temperature, moisture, etc.) display cyclic changes with a period of approximately $24 \mathrm{~h}$. The cycling feature of these factors has fundamental influences on organism health and survival. Most organisms, from single-celled to complex organisms, have evolved internal circadian clock systems that operate in concert physiologically and behaviorally, which enable these organisms to adapt to the environment [1]. A number of representative species across different kingdoms are employed as models in circadian 
clock studies, including cyanobacteria (Synechococcus elongatus); filamentous fungi (Neurospora crassa); unicellular algae (Gonyaulax polyedra); coral (Acropora digitifera); insects, such as fruit flies (Drosophila melanogaster), honeybees (Apis mellifera) and monarch butterflies (Danaus plexippus); plants (Arabidopsis thaliana); mollusks (Bulla gouldiana); fish; birds; rodents and humans [2-8].

At the molecular level, eukaryotic circadian clocks consist of positive and negative components which comprise a set of interplaying clock proteins that act in a highly conserved mechanism. The positive elements are transcription factors that activate transcription of the clock genes. In turn, the clock genes encode negative elements that repress the function of the positive elements. After proteolytic degradation of the clock proteins, the positive elements bind to the promoters of clock genes and re-activate their transcription. Therefore, positive and negative elements constitute negative feedback loops to constitute the circadian oscillators to generate molecular and cellular rhythms, at the transcriptional and translational levels (Figure 1). For instance, in Neurospora, the positive elements are WHITE COLLAR 1 (WC-1) and WC-2, and the negative element is FREQUENCY (FRQ) [2]. In mammals, including humans, the positive elements include BMAL1, CLOCK and RORA, and the negative elements include period proteins (PER1, 2), cryptochrome proteins (CRY1, 2) and REV-ERB $\alpha$ [3]. The circadian clocks operate non-orthologously in a highly conserved manner through transcriptional/translational negative feedback loops, despite phylogenetic differences between circadian clock genes in different species. The positive and negative elements in different organisms are listed in Figure 1. The positive elements also rhythmically drive the expression of clock-controlled genes (ccgs) as circadian output, at the transcriptional level.

Figure 1. The components of circadian clock oscillators. Circadian clock oscillators are composed of positive elements and circadian clock proteins. The positive elements can bind to the promoter regions of the clock genes and mediate the transcription. Circadian clock proteins feed back to repress the functions of positive elements. The positive and negative elements together constitute negative feedback loops at transcription-translational levels. The species names are abbreviated as Nc: Neurospora crassa; Mm: mammals; Dm: Drosophila melanogaster; At: Arabidopsis thaliana. The circadian input and output are not shown.

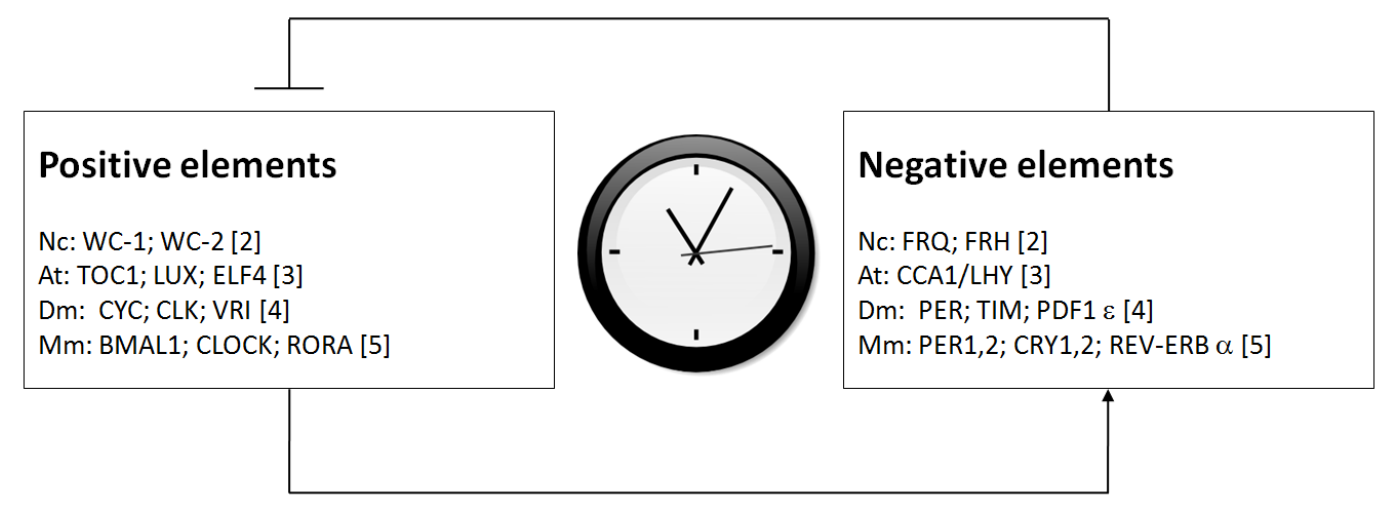

Post-transcriptional regulation is responsible for the regulation in mRNA levels, protein levels as consequence, and diversity of protein isoforms. In addition to transcriptional and translational regulation, epigenetic, post-transcriptional and post-translational controls are emerging as crucial 
modulators of circadian clocks $[9,10]$. Though the core circadian system has concentrated on transcriptional control, it has been apparent that substantial regulation is achieved after transcription. In eukaryotes, approximately $1 \%-10 \%$ genes are subjected to circadian control directly or indirectly [6,11]. Koike et al. reported in a genome-wide study that only $\sim 1 / 5$ of the mRNAs that display rhythmicity in abundance are directly driven by transcription, which suggests that post-transcriptional mechanisms including RNA splicing, polyadenylation, or mRNA stability are essential layers for generation of gene expression rhythmicity [12-16]. Ribonucleoprotein (RNP) complexes play important roles in all aspects of mRNA processing, surveillance, metabolism and turnover at the post-transcriptional level [17,18]. Recently, a number of ribonucleoproteins have been implicated in circadian clocks by regulation of transcription, transcript processing, transport, surveillance and turnover of the circadian gene transcripts [19-25]. Herein, we review the recent findings on post-transcriptional regulation of circadian clocks by RNP complexes.

\section{Regulation of mRNA Splicing}

Alternative splicing is a major cause of mRNA variability and protein diversity in eukaryotes. The process of intron excision occurs in a RNP complex known as a spliceosome. The spliceosome is one of the largest complexes in the cell, and it is composed of $\sim 200$ snRNPs and five snRNAs [26]. Accumulating data suggest that mRNA splicing plays an important role in controlling circadian clock. However, whether splicing is under the control of a circadian clock is lacking substantial evidence.

In Drosophila, modulation of circadian gene expression might contribute to the seasonal adaption of activity patterns. Drosophila exhibits increased mid-day activity on cold days and is more active during the cooler nighttime on hot days. The Drosophila clock gene Per contains intron 8 (dmpi8) in its 3'UTR region, and perturbation of dmpi8 splicing leads to increased accumulation of per transcripts, possibly due to an alteration in the stability of the mRNA [27,28]. Splicing of dmpi8 is inhibited upon light exposure or temperature increase. In addition, it is temporally controlled by the circadian clock mutation in no-receptor potential-A (norpA), which encodes phospholipase C (PLC/NORPA), and has been reported to cause the over-accumulation of spliced per transcripts and an advanced evening activity profile [27,28].

The circadian clock has a property, which enables to maintain its periodicity in spite of daily changes of environmental temperature, which is defined as temperature compensation [29-32]. In Neurospora, the clock genes $w c-2$ and frq express splice variants; the splicing of $f r q$ has been studied extensively [29-36]. Mutation of $w c-2$ at $3484 \mathrm{nt}$, which is located in the midst of a lariat consensus sequence, led to decrease in frq mRNA and FRQ protein levels in the dark [33]. frq can be transcribed into eight splice variants in total, and these transcripts can be classified into two groups according to whether they harbor the 6th intron (I-6) [33,37]. The transcripts with I-6 encode a large-sized FRQ protein isoform (1-FRQ, large FRQ), while the transcripts without I-6 encode a small-sized FRQ isoform (s-FRQ, small FRQ). s-FRQ lacks the $N$-terminal fragment (99aa) due to the use of a different initiation codon than that of l-FRQ [29,30,33]. The expression of 1-FRQ and s-FRQ is controlled by temperature. The ratio of s-FRQ/1-FRQ is increased at a low temperature and decreased at a high temperature. The expression of 1-FRQ inversely correlates with that of s-FRQ [31-34]. Removal of either s-FRQ or 1-FRQ impairs temperature compensation of the circadian clock [30,35], which 
demonstrates that splicing of frq mRNA is critical for the clock temperature compensation in Neurospora.

In the Arabidopsis circadian clock, the core gene $C C A 1$ produces a splice variant $C C A 1 \beta$ that is associated with low temperature responses [38].

A few of splicing factors have been identified to regulate the expression of clock genes and clock phenotypes as a consequence. In Arabidopsis, SNW/Ski-interacting protein (SKIP), a splicing factor and component of the spliceosome, is involved in the post-transcriptional regulation of circadian clock genes in Arabidopsis. SKIP interacts with the spliceosomal splicing factor Ser/Arg-rich protein 45 to regulate pre-mRNA splicing of clock genes, such as PSEUDORESPONSE REGULATOR7 (PRR7) and $P R R$ 9. Mutation in SKIP lengthens the circadian period in a temperature-sensitive manner and affects the circadian clock's response to light input and the sensitivity of the circadian clock to light resetting. In comparison with the wild type gene, the skip- 1 mutant exhibits a comparable circadian period at $27{ }^{\circ} \mathrm{C}$ but a $\sim 3.5 \mathrm{~h}$ longer period at $17{ }^{\circ} \mathrm{C}$, which suggests that SKIP is also required for circadian clock temperature compensation [39]. These facts point to a possibility that mRNA splicing might be a common mechanism in controlling the temperature compensation of circadian clocks in different lineages.

Arabidoposis SPLICEOSOMAL TIMEKEEPER LOCUS1 (STIPL1), a putative RNA-binding protein, is a homolog of the spliceosomal proteins TFP11 (Homo sapiens) and Ntr1p (Saccharomyces cerevisiae) [40]. Mutation in STIPL1 results in a long circadian period. STIPL1 regulates the splicing of the mRNAs of clock genes CCA1, LHY, PRR9, GI, and TOC1 [40]. In Arabidopsis and Drosophila, PRMT5 (PROTEIN ARGININE METHYL TRANSFERASE 5) has also been implicated in regulating the splicing of circadian clock genes, most likely by modifying the methylation status of the Sm spliceosomal proteins [41,42]. More interestingly, PRMT5 is a clock-controlled gene [41], which suggests that the circadian clock might govern the alternative splicing of downstream genes through PRMT5. In contrast, the expression of both SKIP and STIPL1 showed no circadian rhythmicity. It will be intriguing to know whether other core splicing factors are under the circadian control or not.

The RNA-binding protein NONO (also called p54nrb in human and NonO in mouse) contains two RRM domains and a HTH domain, which suggests that it has dual DNA/RNA-binding activities. NONO is localized in the nuclear domains termed paraspeckles in the nucleolus [43,44], and it binds to the 3'end of U5 snRNA stem 1b, which is associated with both the spliceosome and U4/U6.U5 tri-snPNP, suggesting its role in splicing regulation [45]. The $N$-terminus of p54nrb/NonO exhibits high affinity to a set of RNA targets, including $\beta$-globin pre-mRNA, tumor necrosis factor $\alpha$ mRA and the intronic pyrimidine-rich sequence in $\beta$-tropomyosin pre-mRNA $[43,45]$.

Disruption of nonA, the Drosophila homolog of NONO, confers hyperactivity and mild arrhythmicity. Moreover, the mRNA expression of the clock gene timeless is significantly decreased and its rhythmicity is dampened by nonA disruption [45]. NONO can bind to the clock protein PER. Although NONO showed constant expression, its binding to clock protein PER1 displayed circadian rhythmicity [46]. In mice, loss of NONO leads to increased cell proliferation and decreased senescence. NONO binds to the p16-Ink4A cell cycle checkpoint gene and potentiates its circadian activation in a PER protein-dependent fashion, and lack of NONO results in defective wound repair $[47,48]$. NONO is also involved in cell cycle regulation by binding to PER; the two proteins act together as transcription factors for p16-Ink4A, which is a regulator of the mitogen-responsive 
retinoblastoma pathway and one of the key cellular components regulating senescence [48]. Depletion of either NONO or PER led to abolishment circadian expression of p16-Ink4A and elimination of the circadian cell cycle gating, suggesting that PER and NONO are responsible for the coupling of cell cycle to the circadian clock. However, these functions of NONO seem to play a role in transcriptionally regulating the expression of $p 16-\operatorname{Ink} 4 \mathrm{~A}$, instead of alternative splicing.

\section{Control of mRNA Polyadenylation}

The eukaryotic poly(A) tail length at an mRNA 3' end is under cellular control throughout the lifespan of the mRNA. The regulation of mRNA poly(A) tail length likely serves two major purposes: to control mRNA translation and to control mRNA turnover [49]. Intriguingly, Kojima et al. recently reported that the mRNA polyadenylation pattern of a substantial part of the transcriptome is under circadian control [25]. The CPEB (cytoplasmic polyadenylation element-binding protein) proteins bind to cytoplasmic polyadenylation elements (CPEs) in mRNA 3'UTR regions and recruit the polyadenylation complex encompassing the cytoplasmic poly(A) polymerase GLD2 and other related proteins. GLD2 and related proteins promote polyadenylation-induced translation [50]. In mice liver, the mRNA levels of Cpeb2, Cpeb4, Parn and Gld2 are under circadian control, and these genes have been proposed to be responsible for the rhythmicity of transcriptome-scale polyadenylation [25].

Nocturnin (Noc, also called Ccrn 4 l [carbon catabolite repression 4-like]) is a member of the CCR4 deadenylase family, and it encodes a putative deadenylase that degrades mRNA poly(A) tails. Noc is a clock-controlled gene with peak levels during the night [51-55]. The promoter region of Noc harbors an E-box, a cyclic AMP response element (CRE) and a photoreceptor-conserved element II (PCE II), all of which are under circadian control [52,56,57]. Noc is an important mediator of lipid metabolism, adipogenesis, glucose homeostasis, inflammation and osteogenesis [53,58]. In the sponge Suberites domuncula, Nocturnin regulates the deadenylation of the glycogenin mRNA, which encodes a key metabolic enzyme for carbohydrate/glycogen metabolism. Nocturnin levels are high in the dark and low in the light, while glycogenin shows an opposite diurnal expression pattern [59].

Nitric oxide (NO) is a signaling molecule that regulates a diverse group of physiological and pathophysiological activities in cardiovascular, nervous and immunological systems. iNOS (inducible nitric oxide synthase) is a member of the nitric oxide synthase enzyme family, and it catalyzes the synthesis of NO. iNOS is induced by immunostimulatory cytokines, bacterial products or infection [60]. In mice, depletion of Nocturnin results in the destabilization of the $i N O S$ mRNA and the impairment of the nighttime peak profile of hepatic iNOS mRNA, which suggests that the iNOS mRNA might be a target of Noc [61]. It is possible that Nocturnin serves to deadenylate iNOS mRNAs, which further leads to destabilization.

In Neurospora, FRH (FRQ-interacting RNA helicase) plays diverse roles in the regulation of the circadian clock [62-68]. FRH is the counterpart of yeast Mtr4p/Dob1p, which encodes a DexH RNA helicase. In yeast, Mtr4p associates with the Trf4-Air2 heterodimer to form the TRAMP (Trf4/Trf5-Air1/Air2-Mtr4 Polyadenylation) complex, which is an activating cofactor for the exosome. TRAMP promotes the 3'-5' exoribonuclease activity of the exosome, which is responsible for mRNA surveillance and turnover [69-72]. Knockdown of frh in Neurospora has been shown to result in a dramatically slow growth rate and abolishment of conidial rhythmicity [62]. FRQ and FRH form the 
FRQ-FRH complex (FFC), which binds directly to frq mRNA. In an frh knockdown strain, the frq transcripts are more stable and contain significantly longer poly(A) tails compared to wild type, which implies that FRH participates in the regulation of mRNA polyadenylation [63]. FRH might mediate mRNA poly(A) tail length through its partners Trf4 and Trf5 (Topoisomerase one-requiring function 4/5) that are non-canonical poly(A) polymerases $[70,73,74]$, which remains to be further investigated.

Although genome-wide experiments have highlighted the significance of regulation of mRNA poly(A) tail length in the circadian clock, the detailed mechanisms remain unclear. It will be important to address whether and how these RNPs, including NOC and FRH, regulate the poly(A) tail length of clock gene transcripts. In addition, the regulation of polyadenylation occurs in both the nucleus and the cytoplasm, which has functional discrepancy and consequence. It is necessary to elucidate where these factors exert their functions.

\section{4. mRNA Quality Control}

Surveillance mechanisms take place in both the nucleus and cytoplasm to eliminate incorrectly processed transcripts and terminate erroneous gene expression [75,76]. Coupled with as well as following transcription, mRNAs undergo a complicated chain of processing that includes capping, splicing and polyadenylation. Aberrant RNAs can arise from any of these events.

In the nucleus, the exosome is an exoribonuclease complex responsible for rRNA and tRNA biogenesis as well as mRNA surveillance $[69,70]$. Neurospora FRH is the homolog of yeast Mtr4p, a component of the TRAMP complex, which serves as an activating cofactor of the exosome for the degradation of aberrant RNAs via the nuclear RNA surveillance pathway [69,70]. However, whether FRH mediates the elimination of aberrant clock gene transcripts remains unknown.

The nonsense-mediated decay (NMD) pathway is a translation-coupled mRNA surveillance system that typically degrades transcripts bearing premature termination codons (PTCs), thus preventing the translation of unnecessary or aberrant transcripts [77-79]. In addition to its role in quality control, NMD plays a less clear role in regulating the abundance of normal transcripts $[80,81]$.

UPF1 is an RNA helicase that plays a central role in nonsense-mediated mRNA decay (NMD). In Neurospora, the mutant strain period-6 (prd-6), which bears a mutation in upfl, exhibits a shortened circadian period (approximately $19 \mathrm{~h}$ ) and an abnormal temperature compensation of the circadian clock [82].

In Arabidopsis, defects in NMD have been reported to cause impairment in development, seed size control and responses to stress, injury and viral infection [83,84]. In Arabidopsis, AtGRP7 (glycine-rich RNA-binding protein) and AtGRP8 are two nuclear RRM (RNA-binding motif) domain-containing RBPs, and $A t \mathrm{GRP7}$ is a clock gene that is also regulated by the circadian clock $[85,86]$. Overexpression of either AtGRP7 or AtGRP8 leads to the aberrant accumulation of Atgrp7 or Atgrp8 transcripts with PTCs in an NMD-dependent fashion [85-87]. AtGRP7 exhibits a variety of physiological functions, including the promotion of floral transition, plant immunity, growth and stress tolerance. Both atGTP7 and atGRP8 are RNA-binding proteins with a single RRM domain and a glycine-rich $C$-terminus. AtGRP7 controls mRNA processing, mRNA folding and transport of downstream genes, and it plays a role in the circadian output and the regulation of several transcripts involved in stress responses $[88,89]$. 
NMD regulates $\sim 10 \%$ of all cellular mRNAs. In addition to its role in elimination of PTC-containing transcripts, NMD has been implicated in the regulation of other types of transcripts, for instance, mRNAs with upstream open reading frames in their 5'UTRs and mRNAs harboring extended 3'UTRs due to aberrant polyadenylation site usage [75]. Therefore, it is critical to elucidate how NMD affects the circadian clocks at the molecular level.

\section{5. mRNA Nuclear Transport}

Appropriately processed mRNAs are transported from the nucleus to the cytoplasm for subsequent translation, under the control of a series of RBPs and RNP complexes [90]. Cold-inducible RNA-binding protein (CIRP) is a glycine-rich RNA-binding protein harboring an RNA recognition motif (RRM), whose expression can be induced by a mild decrease in temperature [91,92]. In cultured fibroblasts, the rhythmicity of CIRP expression is controlled by temperature cycles instead of circadian oscillators. In a circadian clock, CIRP is necessary for the maintenance of high-amplitude circadian gene expression. CIRP binds to the mRNAs of a number of clock genes and clock-associated genes, including CLOCK, Sirtuin 1 (SIRT1), PERIOD 3 (PER3), NCOR1 and ROR $\alpha$. It has been shown that in CIRP-deficient cells, the Clock mRNA abundance is only moderately changed in nuclei, while the CLOCK protein level is significantly reduced in cytoplasm [91], which suggests that CIRP mediates the export of clock transcripts. CIRP might also regulate the compensation of circadian amplitude at low temperatures in mammals.

FMR1 and FRH are another two proteins that might be potentially implicated in the regulation of transport of clock gene mRNAs.

Fragile $\mathrm{X}$ syndrome, resulting from loss-of-function mutations that abolish the expression of the X-linked gene FMR1 (Fragile X mental retardation 1, also called DFXR), is the most common form of inherited mental retardation in humans [19]. FMRl encodes an RNA-binding protein that contains two ribonucleoprotein $\mathrm{K}$ homology domains ( $\mathrm{KH}$ domains) and an arginine- and glycine-rich domain (RGG box) [19]. FMR1 plays an important role in synaptogenesis and axonal arborization [93]. In addition, FRM1 has been implicated in the regulation of the circadian clock [93-96]. In Drosophila, flies homozygous for $d f m r 1$ deletion displayed erratically timed, short bouts of relatively high activity. It has also been reported that, in constant darkness, the expression of PER and TIM was delayed, and the amplitude of PER was reduced [93]. The expression of cAMP response element-binding protein (CREB), a known clock-controlled gene, was also reduced in fmrl mutants, which suggests a role for FRMR1 in regulating both circadian oscillation and output [94].

There are two FMR1 paralogs in mice, fragile $X$ related gene 1 and 2 (FXR1 and FXR2), which exert their functions in mRNA transportation and translation [95]. Fmr1/Fxr2 double knockout (KO) and Fmrl KO/Fxr2 heterozygous mice exhibit arrhythmic activity in a light-dark (LD) cycle, and Fmr1 or Fxr 2 KO mice display shorter periods of free-running locomotor activity in total darkness (DD) [95]. The expression levels of a number of clock components, including Bmal1, Cry, Per1 and Per2, are significantly altered in these animals [95]. Whether FMR1 influences the circadian clock by regulating the transport of clock gene mRNAs remains to be determined. 
In a yeast $m t r 4$ mutant, poly(A) + RNAs were shown to be retained in the nucleus, suggesting a role for Mtr4p in regulating mRNA transport [96]. It will be interesting to investigate whether FRH, the counterpart of Mtr4p in Neurospora, mediates the transport of clock gene mRNAs.

\section{Translational Regulation}

In mouse liver, $20 \%$ of soluble proteins are subject to circadian control [97]. However, half of these genes do not exhibit rhythmic steady-state mRNA levels, which suggests that translational control also contributes to the generation of gene expression rhythmicity [97,98]. Recently, it has been shown that the mRNAs, including Eif4e, Eif4g1, Eif4a2, Eif4b, Eif4ebp1 and Eif4ebp3, which encode factors involved in translation initiation, are under circadian control. Moreover, the phosphorylation of EIF4G, EIF4B, 4EBP1 and ribosomal protein (RP) S6 (RPS6) oscillate in a circadian fashion, which peaks during the night [98]. These findings suggest that the circadian clock controls ribosome biogenesis and gene expression at the translational level.

Drosophila eclosion and locomotor activity are controlled by its circadian clock. LARK negatively regulates eclosion; decreased LARK levels account for an early eclosion phenotype, while increased LARK levels correspond to a late eclosion phenotype [99]. lark encodes a protein with two RNA recognition motifs (RRMs) and a retroviral-type (RT) zinc finger domain. LARK has been recently found to associate with and stabilize fragile X mental retardation protein (FMRP). The two proteins act together to regulate eye development and circadian behavior. In Drosophila, it has been shown that overexpression of LARK conferred arrhythmicity and a reduction of $d f m r 1$ expression can mostly rescue the arrhythmic phenotype, which suggests that FMRP promotes LARK activity [100]. In mouse suprachiasmatic nuclei (SCN), a tissue, which is an autonomous circadian pacemaker, the level of LARK protein oscillates with a similar phase to PER1 protein although its mRNA shows no rhythmicity [101]. LARK post-transcriptionally regulates Perl expression by binding to the 3'UTR of Per1 mRNA, which further promotes the synthesis of PER1 protein. Knockdown of Lark resulted in short circadian period while overexpression of Lark resulted in prolonged period, which suggests that LARK might regulate circadian clock by controlling the translation of Perl.

Rev-erb $\alpha$ encodes a transcriptional repressor in the positive limb of circadian transcription (Figure 1), which harbors an internal ribosomal entry site (IRES) in its mRNA 5'UTR region [102]. IRES regulates the translation by recruiting ribosomes in a cap-independent manner. PTB is a RNA-binding protein which plays roles in diverse cellular processes, including polyadenylation, mRNA stability and translation initiation. hnRNP Q is a member of hnRNP family, which function to bind pre-mRNAs and facilitate their processing of mRNAs. Rev-erb $\alpha$ mRNA has an internal ribosomal entry site (IRES) in its 5'-UTR, which can be bound by PTB and hnRNP Q; PTB and hnRNP Q function to enhance the translation of Rev-erb $\alpha$ through the IRES element [102]. hnRNP Q is also an IRES trans-acting factor of mouse clock gene Period 1 (Perl), and the binding between mhnRNP Q and mPerl mRNA is in a rhythmic fashion. Knockdown of mhnRNP Q caused a decrease in PER1 protein levels and a slight delay in mPER1 expression without change in mRNA abundance [103].

hnRNP Q modulates the translation of AANAT protein levels in a similar way. Serotonin $N$-acetyltransferase (arylalkylamine $N$-acetyltransferase (AANAT) is the key enzyme in melatonin 
synthesis regulated by the circadian clock and AANAT mRNA contains an IRES element within its 5'UTR. The $68-\mathrm{kDa}$ hnRNP Q binds specifically to AANAT IRES element; hnRNP enhances the translation and contributes to AANAT rhythmicity at the protein level [104]. These data suggest that hnRNP Q plays an important role in translational control of melatonin synthesis. In addition, hnRNP Q is a spliceosomal component, however, whether hnRNP Q mediates mRNA splicing of clock genes remains elusive.

Bioluminescence is generated by a luciferase-catalyzed reaction involving the substrate luciferin. Binding and stabilization of luciferin by luciferin-binding protein (LBP) is also necessary for the bioluminescence. The bioluminescence in Gonyaulax polyedra is controlled by circadian clock, which displays a night phase in vivo. In contrast to its constant mRNA level, the LBP protein abundance oscillates between day and night with a 10-fold circadian variation [105]. Circadian-controlled translational regulator (CCTR) rhythmically binds to $l b p$ mRNA at the 22-nt UG repeat-containing region of its $3^{\prime} \mathrm{UTR}$ and represses the translation of target transcripts [106]. In the green alga Chlamydomonas reinhardtii, clock-controlled RNA-binding protein (CHLAMY 1) is an analog of Gonyaulax CCTR. CHLAMY 1 binds specifically to the 3'UTRs of several mRNAs and recognizes them all via a common cis-acting element [107]. LBP is an example that evidences the role of translational control in generating the circadian rhythmicity.

The ribosome is a large ribonucleoprotein complex that functions as the translational machinery of the cell, and translation efficiency is essential for controlling protein translation, conformation and function [108]. The translation rate depends on the codon usage since it is more efficient for the transfer RNA (tRNAs) to recognize preferential codons and carry the corresponding amino acid to the ribosome for peptide synthesis. The process of translation of certain clock proteins is strictly controlled by the genetic codon usage. Two independent studies recently revealed that in Neurospora and cyanobacteria, non-preferential codon usage for the clock genes (frq in N. crassa and KaiBC in S. elongatus) determines the translation, conformation or function of the clock proteins $[109,110]$. Optimization of the genetic codon usage in Neurospora frq gene, which uses non-optimal codons resulted in severe impairment of the conidiation rhythmicity. More interestingly, it led to changes in phosphorylation profile, conformation and function of FRQ protein [109]. In the cyanobacterium $S$. elongates, optimization of $\mathrm{KaiBC}$ genes resulted in promoted amplitude at low temperatures. However, at low temperatures, the strain with optimization of KaiBC exhibits a slower growth rate [110], which suggests that codon usage is essential for the adaption to the ambient conditions. In humans, the synonymous substitution polymorphism T2434C in the clock gene PERl has been reported to be associated with extreme diurnal preference. C2434 was more frequently found in subjects with extreme morning preference than in subjects with extreme evening preference [111]. Whether the T2434C polymorphism affects mRNA stability or translation rate is unknown.

\section{Regulation of mRNA Turnover}

The steady-state level of mRNA in a cell is determined by both its production and lifespan. Regulation of mRNA turnover is a critical event during post-transcriptional processing as alteration in mRNA abundance may change the amount of the corresponding protein. mRNA turnover is controlled by a series of factors in a stepwise fashion, encompassing decapping, deadenylation and degradation [112]. 
In the cytoplasm, the exosome complex regulates the $3^{\prime}-5^{\prime}$ degradation of mRNAs. Of the 10 subunits of the exosome, RRP44/Dis3 is the catalytic subunit with nuclease activity $[113,114]$. In Neurospora, knockdown of rrp44 resulted in stabilization of frq mRNA. In addition, RRP44 regulates the expression of a set of clock-controlled genes either directly or indirectly. The conidial rhythmicity was abolished and the longer circadian periods of frq mRNA and FRQ protein were significantly longer in rrp44-silenced strain [63].

AU-rich elements (AREs) are located in the 3'UTR of many mRNAs and found in short-lived transcripts of a diverse group of genes, such as cytokines, proto-oncogenes, growth factors, and cell cycle regulators [115]. AREs generally serve as binding sites for AU-binding proteins (AUBPs), which cause deadenylation, decapping and degradation of the target transcripts. Therefore, AUBPs are known to have mRNA decay-promoting properties. Butyrate response factor-1 (BRF1, also called ZFP36L1/Tis11b) is an AUBP that possesses two zinc-finger domains in tandem [116]. BRF1 is expressed in a circadian fashion [116], which suggests that it might be involved in the post-transcriptional regulation of gene expression rhythmicity. Analogously, although the Neurospora frq gene contains no canonic ARE, the 3'UTR region of frq determines its transcript decay rate [63], both cis-elements and trans-factors might be involved in regulating the decay of frq mRNA. hnRNP proteins are involved in the regulation of mRNA stability. In mammals, hnRNP Q, hnRNP L and hnRNP R bind to AANAT mRNA 3'UTR region and destabilize the transcripts; hnRNP Q, hnRNP L and hnRNP R are necessary for maintaining the normal rhythm of melatonin [117]. hnRNP Q also binds to both the mRNA $5^{\prime}$ and $3^{\prime}$ UTR regions of mouse clock gene Period3, to regulate the translation and mRNA decay respectively [118]. There are several known pathways that control the mRNA turnover, which are $3^{\prime} \rightarrow 5^{\prime}$ exonucleolytic decay, 5' $\rightarrow 3^{\prime}$ exonucleolytic decay and endonuclease cleavage, respectively $[112,115]$. It will be critical to elucidate the following pathways coupled to hnRNP proteins to regulate the turnover of clock gene transcripts.

miRNAs (micro RNAs) are small non-coding RNAs with a length of approximately 22 nucleotides that trigger gene silencing. miRNA-mediated gene silencing is an important mechanism of gene expression regulation in many eukaryotes through facilitation of mRNA decay or translational repression $[119,120]$. Dicer, an RNase III-type endonuclease, and RISC (RNA-induced silencing complex) are two essential RNP complexes required for miRNA-mediated gene silencing [121]. A number of miRNAs have been implicated in the regulation of circadian clocks in different species [122-128]. It is reasonable to speculate that some miRNAs might be responsible for the generation of circadian rhythmicity at the protein level of a set of genes that are constantly expressed at the mRNA level.

\section{Summary and Outlook}

Eukaryotic genomes encode a large number of RBPs that are involved in the regulation of a variety of biological processes [17,19]. Increasing data have demonstrated that RNP complexes play multiple roles in regulating the expression of circadian clock genes (Figure 2). However, little is known about the roles of RNP complexes in certain steps of mRNA processing, such as mRNA capping/decapping and transport. Post-transcriptional steps are tightly coupled and interwoven, which suggests that ribonucleoprotein complexes constitute highly complex regulatory networks coordinating to sustain circadian clock systems. Some proteins, like hnRNP Q, FRH and NONO, appear to be versatile players 
in multiple post-transcriptional regulations. Relative to the accumulation of evidence suggesting the influence on the circadian phenotypes, the functional details of these RNP/RNP complexes underlying the circadian clocks remain to be intensively investigated. Therefore, it is of significant importance to systematically identify more clock-associated RNPs and elucidate their roles. With the cutting edge technologies, we will be able to identify more RNPs involved in circadian clock and target transcripts in a large scale.

Figure 2. Known RNP factors involved in stepwise controlling of expression of clock genes on the mRNA level. The species names are abbreviated as Nc: Neurospora crassa; Mm: mammals; Dm: Drosophila melanogaster; At: Arabidopsis thaliana; Cr: Chlamydomonas reinhardtii; Gp: Gonyaulax polyedra; Se: Synechococcus elongates; Sd: Suberites domuncula; Xl: Xenopus laevis.

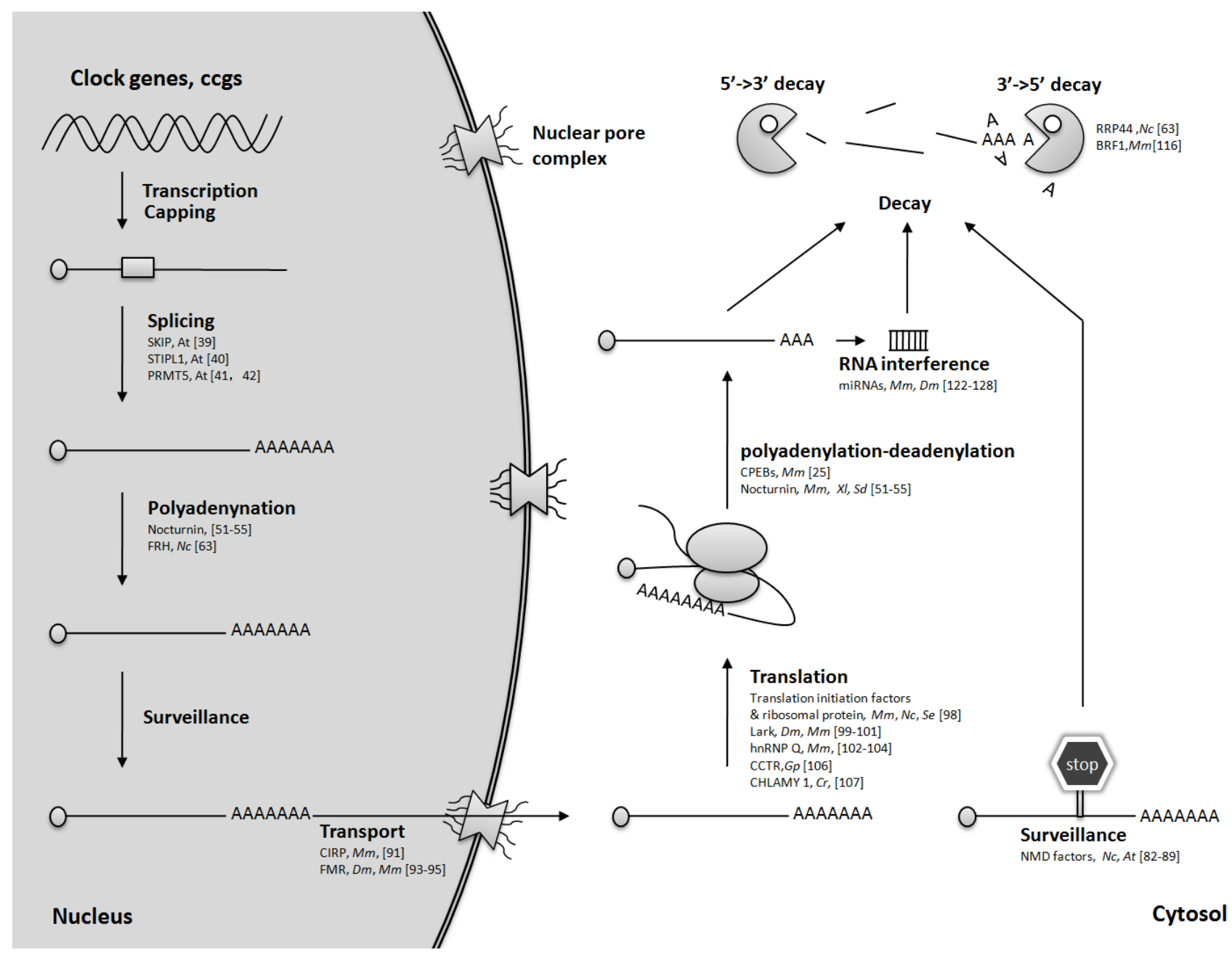

It is not surprising that these networks might be involved in temperature compensation, which is an important property of any circadian clock [30]. Moreover, comprehensive modulation of circadian clocks by nucleoprotein complexes is likely to account for the fine regulation of the circadian systems under various conditions and stresses. Further investigation of the RNP roles in these issues will expand our knowledge of both the circadian clock mechanisms and the physiological relevance of circadian clocks. In addition, studies of the roles of SNPs in circadian clocks will no doubt help in revision of the basic model of the generation of circadian rhythms. 


\section{Acknowledgments}

This work was supported by the National 973 Program of China (No. 2011CB711000 and 2012CB947600), the National Natural Science Foundation of China (No. 31071122 and No. 31171119), the Fundamental Research Funds for the Central Universities of China (11lgzd08), the Research Fund for the Doctoral Program of Higher Education of China (20110171110030), Scientific Research Foundation for Returned Scholars, Ministry of Education of China ([2012]1707) and New Century Training Program Foundation for the Talents by the State Education Commission (NCET-12-0566).

\section{Conflict of Interest}

The authors declare no conflict of interest.

\section{References}

1. Duguay, D.; Cermakian, N. The crosstalk between physiology and circadian clock proteins. Chronobiol. Int. 2009, 26, 1479-1513.

2. Dunlap, J.C.; Loros, J.J. The neurospora circadian system. J. Biol. Rhythms. 2004, 19, 414-424.

3. Nagel, D.H.; Kay, S.A. Complexity in the wiring and regulation of plant circadian networks. Curr. Biol. 2012, 22, R648-R657.

4. Ozkaya, O.; Rosato, E. The circadian clock of the fly: A neurogenetics journey through time. Adv. Genet. 2012, 77, 79-123.

5. Albrecht, U.; Eichele, G. The mammalian circadian clock. Curr. Opin. Genet. Dev. 2003, 13, 271-277.

6. Bell-Pedersen, D.; Cassone, V.M.; Earnest, D.J.; Golden, S.S.; Hardin, P.E.; Thomas, T.L.; Zoran, M.J. Circadian rhythms from multiple oscillators: Lessons from diverse organisms. Nat. Rev. Genet. 2005, 6, 544-556.

7. Shoguchi, E.; Tanaka, M.; Shinzato, C.; Kawashima, T.; Satoh, N. A genome-wide survey of photoreceptor and circadian genes in the coral, Acropora digitifera. Gene 2013, 515, 426-431.

8. Chabot, J.R.; Pedraza, J.M.; Luitel, P.; van Oudenaarden, A. Stochastic gene expression out-of-steady-state in the cyanobacterial circadian clock. Nature 2007, 450, 1249-1252.

9. Feng, D.; Lazar, M.A. Clocks, metabolism, and the epigenome. Mol. Cell 2012, 47, 158-167.

10. Kawazoe, R.; Mahan, K.M.; Venghaus, B.E.; Carter, M.L.; Herrin, D.L. Circadian regulation of chloroplast transcription in Chlamydomonas is accompanied by little or no fluctuation in RPOD levels or core RNAP activity. Mol. Biol. Rep. 2012, 39, 10565-10571.

11. Lowrey, P.L.; Takahashi, J.S. Mammalian circadian biology: Elucidating genome-wide levels of temporal organization. Annu. Rev. Genomics Hum. Genet. 2004, 5, 407-441.

12. Zwiebel, L.J.; Hardin, P.E.; Liu, X.; Hall, J.C.; Rosbash, M. A post-transcriptional mechanism contributes to circadian cycling of a per-beta-galactosidase fusion protein. Proc. Natl. Acad. Sci. USA 1991, 88, 3882-3886.

13. So, W.V.; Rosbash, M. Post-transcriptional regulation contributes to Drosophila clock gene mRNA cycling. EMBO J. 1997, 16, 7146-7155. 
14. Zhang, L.; Weng, W.; Guo, J. Posttranscriptional mechanisms in controlling eukaryotic circadian rhythms. FEBS Lett. 2011, 585, 1400-1405.

15. Koike, N.; Yoo, S.H.; Huang, H.C.; Kumar, V.; Lee, C.; Kim, T.K.; Takahashi, J.S. Transcriptional architecture and chromatin landscape of the core circadian clock in mammals. Science 2012, 338, 349-354.

16. Menet, J.S.; Rodriguez, J.; Abruzzi, K.C.; Rosbash, M. Nascent-Seq reveals novel features of mouse circadian transcriptional regulation. ELife Sci. 2012, 1, e00011.

17. Dreyfuss, G.; Kim, V.N.; Kataoka, N. Messenger-RNA-binding proteins and the messages they carry. Nat. Rev. Mol. Cell. Biol. 2002, 3, 195-205.

18. Glisovic, T.; Bachorik, J.L.; Yong, J.; Dreyfuss, G. RNA-binding proteins and post-transcriptional gene regulation. FEBS Lett. 2008, 582, 1977-1986.

19. Gao, F.B. Understanding fragile X syndrome: Insights from retarded flies. Neuron 2002, 34, 859-862.

20. Harms, E.; Kivimae, S.; Young, M.W.; Saez, L. Posttranscriptional and posttranslational regulation of clock genes. J. Biol. Rhythms 2004, 19, 361-373.

21. Cibois, M.; Gautier-Courteille, C.; Legagneux, V.; Paillard, L. Post-transcriptional controlsAdding a new layer of regulation to clock gene expression. Trends Cell Biol. 2010, 20, 533-541.

22. Kojima, S.; Shingle, D.L.; Green, C.B. Post-transcriptional control of circadian rhythms. J. Cell Sci. 2011, 124, 311-320.

23. Staiger, D.; Green, R. RNA-based regulation in the plant circadian clock. Trends Plant Sci. 2011, 16, 517-523.

24. Staiger, D.; Koster, T. Spotlight on post-transcriptional control in the circadian system. Cell. Mol. Life Sci. 2011, 68, 71-83.

25. Kojima, S.; Sher-Chen, E.L.; Green, C.B. Circadian control of mRNA polyadenylation dynamics regulates rhythmic protein expression. Genes Dev. 2012, 26, 2724-2736.

26. Wahl, M.C.; Will, C.L.; Luhrmann, R. The spliceosome: Design principles of a dynamic RNP machine. Cell 2009, 136, 701-718.

27. Majercak, J.; Chen, W.F.; Edery, I. Splicing of the period gene 3'-terminal intron is regulated by light, circadian clock factors, and phospholipase C. Mol. Cell. Biol. 2004, 24, 3359-3372.

28. Low, K.H.; Chen, W.F.; Yildirim, E.; Edery, I. Natural Variation in the Drosophila melanogaster Clock Gene Period Modulates Splicing of Its 3'-Terminal Intron and Mid-Day Siesta. PLoS One 2012, 7, e49536.

29. Liu, Y.; Garceau, N.Y.; Loros, J.J.; Dunlap, J.C. Thermally regulated translational control of FRQ mediates aspects of temperature responses in the neurospora circadian clock. Cell 1997, 89, 477-486.

30. Liu, Y.; Merrow, M.; Loros, J.J.; Dunlap, J.C. How temperature changes reset a circadian oscillator. Science 1998, 281, 825-829.

31. Neiss, A.; Schafmeier, T.; Brunner; M. Transcriptional regulation and function of the Neurospora clock gene white collar 2 and its isoforms. EMBO Rep. 2008, 9, 788-794.

32. Diernfellner, A.; Colot, H.V.; Dintsis, O.; Loros, J.J.; Dunlap, J.C.; Brunner, M. Long and short isoforms of Neurospora clock protein FRQ support temperature-compensated circadian rhythms. FEBS Lett. 2007, 581, 5759-5764.

33. Collett, M.A.; Dunlap, J.C.; Loros, J.J. Circadian clock-specific roles for the light response protein WHITE COLLAR-2. Mol. Cell. Biol. 2001, 21, 2619-2628. 
34. Diernfellner, A.C.; Schafmeier, T.; Merrow, M.W.; Brunner, M. Molecular mechanism of temperature sensing by the circadian clock of Neurospora crassa. Genes Dev. 2005, 19, 1968-1973.

35. Kaldi, K.; Gonzalez, B.H.; Brunner, M. Transcriptional regulation of the Neurospora circadian clock gene wc-1 affects the phase of circadian output. EMBO Rep. 2006, 7, 199-204.

36. Baker, C.L.; Loros, J.J.; Dunlap, J.C. The circadian clock of Neurospora crassa. FEMS Microbiol. Rev. 2012, 36, 95-110.

37. Colot, H.V.; Loros, J.J.; Dunlap, J.C. Temperature-modulated alternative splicing and promoter use in the Circadian clock gene frequency. Mol. Biol. Cell 2005, 16, 5563-5571.

38. Park, M.J.; Seo, P.J.; Park, C.M. CCA1 alternative splicing as a way of linking the circadian clock to temperature response in Arabidopsis. Plant Signal. Behav. 2012, 7, 1194-1196.

39. Wang, X.; Wu, F.; Xie, Q.; Wang, H.; Wang, Y.; Yue, Y.; Gahura, O.; Ma, S.; Liu, L.; Cao, Y.; et al. SKIP is a component of the spliceosome linking alternative splicing and the circadian clock in Arabidopsis. Plant Cell 2012, 24, 3278-3295.

40. Jones, M.A.; Williams, B.A.; McNicol, J.; Simpson, C.G.; Brown, J.W.; Harmer, S.L. Mutation of Arabidopsis SPLICEOSOMAL TIMEKEEPER LOCUS1 Causes Circadian Clock Defects. Plant Cell 2012, 24, 4066-4082.

41. Sanchez, S.E.; Petrillo, E.; Beckwith, E.J.; Zhang, X.; Rugnone, M.L.; Hernando, C.E.; Cuevas, J.C.; Godoy Herz, M.A.; Depetris-Chauvin, A.; Simpson, C.G.; et al. A methyl transferase links the circadian clock to the regulation of alternative splicing. Nature 2010, 468, 112-116.

42. Hong, S.; Song, H.R.; Lutz, K.; Kerstetter, R.A.; Michael, T.P.; McClung, C.R. Type II protein arginine methyltransferase 5 (PRMT5) is required for circadian period determination in Arabidopsis thaliana. Proc. Natl. Acad. Sci. USA 2010, 107, 21211-21216.

43. Shav-Tal, Y.; Zipori, D. PSF and p54nrb/NonO-Multi-functional nuclear proteins. FEBS Lett. 2002, 531, 109-114.

44. Guillaumond, F.; Boyer, B.; Becquet, D.; Guillen, S.; Kuhn, L.; Garin, J.; Belghazi, M.; Bosler, O.; Franc, J.L.; Francois-Bellan, A.M. Chromatin remodeling as a mechanism for circadian prolactin transcription: Rhythmic NONO and SFPQ recruitment to HLTF. FASEB J. 2011, 25, 2740-2756.

45. Peng, R.; Dye, B.T.; Perez, I.; Barnard, D.C.; Thompson, A.B.; Patton, J.G. PSF and p54nrb bind a conserved stem in U5 snRNA. RNA 2002, 8, 1334-1347.

46. Brown, S.A.; Ripperger, J.; Kadener, S.; Fleury-Olela, F.; Vilbois, F.; Rosbash, M.; Schibler, U. PERIOD1-associated proteins modulate the negative limb of the mammalian circadian oscillator. Science 2005, 308, 693-696.

47. Passon, D.M.; Lee, M.; Rackham, O.; Stanley, W.A.; Sadowska, A.; Filipovska, A.; Fox, A.H.; Bond, C.S. Structure of the heterodimer of human NONO and paraspeckle protein component 1 and analysis of its role in subnuclear body formation. Proc. Natl. Acad. Sci. USA 2012, 109, 4846-4850.

48. Kowalska, E.; Ripperger, J.A.; Hoegger, D.C.; Bruegger, P.; Buch, T.; Birchler, T.; Mueller, A.; Albrecht, U.; Contaldo, C.; Brown, S.A. NONO couples the circadian clock to the cell cycle. Proc. Natl. Acad. Sci. USA 2012, 110, 1592-1599.

49. Jacobson, A.; Peltz, S.W. Interrelationships of the pathways of mRNA decay and translation in eukaryotic cells. Annu. Rev. Biochem. 1996, 65, 693-739. 
50. Lin, C.L.; Evans, V.; Shen, S.; Xing, Y.; Richter, J.D. The nuclear experience of CPEB: Implications for RNA processing and translational control. RNA 2010, 16, 338-348.

51. Kowalska, E.; Ripperger, J.A.; Muheim, C.; Maier, B.; Kurihara, Y.; Fox, A.H.; Kramer, A.; Brown, S.A. Distinct Roles of DBHS Family Members in the Circadian Transcriptional Feedback Loop. Mol. Cell. Biol. 2012, 32, 4585-4594.

52. Green, C.B.; Besharse, J.C. Identification of a novel vertebrate circadian clock-regulated gene encoding the protein nocturnin. Proc. Natl. Acad. Sci. USA 1996, 93, 14884-14888.

53. Baggs, J.E.; Green, C.B. Nocturnin, a deadenylase in Xenopus laevis retina: A mechanism for posttranscriptional control of circadian-related mRNA. Curr. Biol. 2003, 13, 189-198.

54. Wang, Y.; Osterbur, D.L.; Megaw, P.L.; Tosini, G.; Fukuhara, C.; Green, C.B.; Besharse, J.C. Rhythmic expression of Nocturnin mRNA in multiple tissues of the mouse. BMC Dev. Biol. 2001, 1, doi:10.1186/1471-213X-1-9.

55. Oishi, K.; Miyazaki, K.; Kadota, K.; Kikuno, R.; Nagase, T.; Atsumi, G.; Ohkura, N.; Azama, T.; Mesaki, M.; Yukimasa, S.; et al. Genome-wide expression analysis of mouse liver reveals CLOCK-regulated circadian output genes. J. Biol. Chem. 2003, 278, 41519-41527.

56. Liu, X.; Green, C.B. A novel promoter element, photoreceptor conserved element II, directs photoreceptor-specific expression of nocturnin in Xenopus laevis. J. Biol. Chem. 2001, 276, $15146-15154$.

57. Li, R.; Yue, J.; Zhang, Y.; Zhou, L.; Hao, W.; Yuan, J.; Qiang, B.; Ding, J.M.; Peng, X.; Cao, J.M. CLOCK/BMAL1 regulates human nocturnin transcription through binding to the E-box of nocturnin promoter. Mol. Cell. Biochem. 2008, 317, 169-177.

58. Hussain, M.M.; Pan, X. Clock regulation of dietary lipid absorption. Curr. Opin. Clin. Nutr. Metab. Care 2012, 15, 336-341.

59. Muller, W.E.; Wang, X.; Grebenjuk, V.A.; Korzhev, M.; Wiens, M.; Schlossmacher, U.; Schroder, H.C. Nocturnin in the demosponge Suberites domuncula: A potential circadian clock protein controlling glycogenin synthesis in sponges. Biochem. J. 2012, 448, 233-242.

60. Aktan, F. iNOS-mediated nitric oxide production and its regulation. Life Sci. 2004, 75, 639-653.

61. Niu, S.; Shingle, D.L.; Garbarino-Pico, E.; Kojima, S.; Gilbert, M.; Green, C.B. The circadian deadenylase Nocturnin is necessary for stabilization of the iNOS mRNA in mice. PLoS One 2011, 6, e26954.

62. Cheng, P.; He, Q.; He, Q.; Wang, L.; Liu, Y. Regulation of the Neurospora circadian clock by an RNA helicase. Genes Dev. 2005, 19, 234-241.

63. Guo, J.; Cheng, P.; Yuan, H.; Liu, Y. The Exosome regulates circadian gene expression in a posttranscriptional negative feedback loop. Cell 2009, 138, 1236-1246.

64. Guo, J.; Cheng, P.; Liu, Y. Functional significance of FRH in regulating the phosphorylation and stability of Neurospora circadian clock protein FRQ. J. Biol. Chem. 2010, 285, 11508-11515.

65. Hunt, S.M.; Thompson, S.; Elvin, M.; Heintzen, C. VIVID interacts with the WHITE COLLAR complex and FREQUENCY-interacting RNA helicase to alter light and clock responses in Neurospora. Proc. Natl. Acad. Sci. USA 2010, 107, 16709-16714.

66. Hunt, S.; Elvin, M.; Heintzen, C. Temperature-sensitive and circadian oscillators of Neurospora crassa share components. Genetics 2012, 191, 119-131. 
67. Shi, M.; Collett, M.; Loros, J.J.; Dunlap, J.C. FRQ-interacting RNA helicase mediates negative and positive feedback in the Neurospora circadian clock. Genetics 2010, 184, 351-361.

68. Belden, W.J.; Lewis, Z.A.; Selker, E.U.; Loros, J.J.; Dunlap, J.C. CHD1 remodels chromatin and influences transient DNA methylation at the clock gene frequency. PLoS Genet. 2011, 7, e1002166.

69. Van Hoof, A.; Lennertz, P.; Parker, R. Yeast exosome mutants accumulate 3'-extended polyadenylated forms of U4 small nuclear RNA and small nucleolar RNAs. Mol. Cell. Biol. 2000, 20, 441-452.

70. LaCava, J.; Houseley, J.; Saveanu, C.; Petfalski, E.; Thompson, E.; Jacquier, A.; Tollervey, D. RNA degradation by the exosome is promoted by a nuclear polyadenylation complex. Cell $\mathbf{2 0 0 5}$, 121, 713-724.

71. Holub, P.; Lalakova, J.; Cerna, H.; Pasulka, J.; Sarazova, M.; Hrazdilova, K.; Arce, M.S.; Hobor, F.; Stefl, R.; Vanacova, S. Air2p is critical for the assembly and RNA-binding of the TRAMP complex and the KOW domain of Mtr4p is crucial for exosome activation. Nucleic Acids Res. 2012, 40, 5679-5693.

72. Fasken, M.B.; Leung, S.W.; Banerjee, A.; Kodani, M.O.; Chavez, R.; Bowman, E.A.; Purohit, M.K.; Rubinson, M.E.; Rubinson, E.H.; Corbett, A.H. Air1 zinc knuckles 4 and 5 and a conserved IWRXY motif are critical for the function and integrity of the Trf4/5-Air1/2-Mtr4 polyadenylation (TRAMP) RNA quality control complex. J. Biol. Chem. 2011, 286, 37429-37445.

73. Sadoff, B.U.; Heath-Pagliuso, S.; Castano, I.B.; Zhu, Y.; Kieff, F.S.; Christman, M.F. Isolation of mutants of Saccharomyces cerevisiae requiring DNA topoisomerase I. Genetics 1995, 141, 465-479.

74. Haracska, L.; Johnson, R.E.; Prakash, L.; Prakash, S. Trf4 and Trf5 proteins of Saccharomyces cerevisiae exhibit poly(A) RNA polymerase activity but no DNA polymerase activity. Mol. Cell. Biol. 2005, 25, 10183-10189.

75. Wagner, E.; Lykke-Andersen, J. mRNA surveillance: The perfect persist. J. Cell Sci. 2002, 115, 3033-3038.

76. Doma, M.K.; Parker, R. RNA quality control in eukaryotes. Cell 2007, 131, 660-668.

77. He, F.; Li, X.; Spatrick, P.; Casillo, R.; Dong, S.; Jacobson, A. Genome-wide analysis of mRNAs regulated by the nonsense-mediated and 5' to 3' mRNA decay pathways in yeast. Mol. Cell 2003, $12,1439-1452$.

78. Rehwinkel, J.; Letunic, I.; Raes, J.; Bork, P.; Izaurralde, E. Nonsense-mediated mRNA decay factors act in concert to regulate common mRNA targets. RNA 2005, 11, 1530-1544.

79. Chang, Y.F.; Imam, J.S.; Wilkinson, M.F. The nonsense-mediated decay RNA surveillance pathway. Annu. Rev. Biochem. 2007, 76, 51-74.

80. Metzstein, M.M.; Krasnow, M.A. Functions of the nonsense-mediated mRNA decay pathway in Drosophila development. PLoS Genet. 2006, 2, e180.

81. Rebbapragada, I.; Lykke-Andersen, J. Execution of nonsense-mediated mRNA decay: What defines a substrate? Curr. Opin. Cell. Biol. 2009, 21, 394-402.

82. Morgan, L.W.; Feldman, J.F. Isolation and characterization of a temperature-sensitive circadian clock mutant of Neurospora crassa. Genetics 1997, 146, 525-530.

83. Jeong, H.J.; Kim, Y.J.; Kim, S.H.; Kim, Y.H.; Lee, I.J.; Kim, Y.K.; Shin, J.S. Nonsense-mediated mRNA decay factors, UPF1 and UPF3, contribute to plant defense. Plant Cell Physiol. 2011, 52, 2147-2156. 
84. Shi, C.; Baldwin, I.T.; Wu, J. Arabidopsis plants having defects in nonsense-mediated mRNA decay factors UPF1, UPF2, and UPF3 show photoperiod-dependent phenotypes in development and stress responses. J. Integr. Plant Biol. 2012, 54, 99-114.

85. Heintzen, C.; Nater, M.; Apel, K.; Staiger, D. AtGRP7, a nuclear RNA-binding protein as a component of a circadian-regulated negative feedback loop in Arabidopsis thaliana. Proc. Natl. Acad. Sci. USA 1997, 94, 8515-8120.

86. Schoning, J.C.; Streitner, C.; Page, D.R.; Hennig, S.; Uchida, K.; Wolf, E.; Furuya, M.; Staiger, D. Auto-regulation of the circadian slave oscillator component AtGRP7 and regulation of its targets is impaired by a single RNA recognition motif point mutation. Plant J. 2007, 52, 1119-1130.

87. Staiger, D.; Zecca, L.; Wieczorek Kirk, D.A.; Apel, K.; Eckstein, L. The circadian clock regulated RNA-binding protein AtGRP7 autoregulates its expression by influencing alternative splicing of its own pre-mRNA. Plant J. 2003, 33, 361-371.

88. Kim, J.S.; Jung, H.J.; Lee, H.J.; Kim, K.A.; Goh, C.H.; Woo, Y.; Oh, S.H.; Han, Y.S.; Kang, H. Glycine-rich RNA-binding protein 7 affects abiotic stress responses by regulating stomata opening and closing in Arabidopsis thaliana. Plant J. 2008, 55, 455-466.

89. Streitner, C.; Hennig, L.; Korneli, C.; Staiger, D. Global transcript profiling of transgenic plants constitutively overexpressing the RNA-binding protein AtGRP7. BMC Plant Biol. 2010, 10, doi:10.1186/1471-2229-10-221.

90. Kelly, S.M.; Corbett, A.H. Messenger RNA export from the nucleus: A series of molecular wardrobe changes. Traffic 2009, 10, 1199-1208.

91. Morf, J.; Rey, G.; Schneider, K.; Stratmann, M.; Fujita, J.; Naef, F.; Schibler, U. Cold-inducible RNA-binding protein modulates circadian gene expression posttranscriptionally. Science 2012, 338, 379-383.

92. Xia, Z.P.; Zheng, X.M.; Zheng, H.; Liu, X.J.; Liu, G.Y.; Wang, X.H. Downregulation of cold-inducible RNA-binding protein activates mitogen-activated protein kinases and impairs spermatogenic function in mouse testes. Asian J. Androl. 2012, 14, 884-889.

93. Inoue, S.; Shimoda, M.; Nishinokubi, I.; Siomi, M.C.; Okamura, M.; Nakamura, A.; Kobayashi, S.; Ishida, N.; Siomi, H. A role for the Drosophila fragile X-related gene in circadian output. Curr. Biol. 2002, 12, 1331-1335.

94. Dockendorff, T.C.; Su, H.S.; McBride, S.M.; Yang, Z.; Choi, C.H.; Siwicki, K.K.; Sehgal, A.; Jongens, T.A. Drosophila lacking dfmr1 activity show defects in circadian output and fail to maintain courtship interest. Neuron 2002, 34, 973-984.

95. Zhang, J.; Fang, Z.; Jud, C.; Vansteensel, M.J.; Kaasik, K.; Lee, C.C.; Albrecht, U.; Tamanini, F.; Meijer, J.H.; Oostra, B.A.; et al. Fragile X-related proteins regulate mammalian circadian behavioral rhythms. Am. J. Hum. Genet. 2008, 83, 43-52.

96. Liang, S.; Hitomi, M.; Hu, Y.H.; Liu, Y.; Tartakoff, A.M. A DEAD-box-family protein is required for nucleocytoplasmic transport of yeast mRNA. Mol .Cell. Biol. 1996, 16, 5139-5146.

97. Reddy, A.B.; Karp, N.A.; Maywood, E.S.; Sage, E.A.; Deery, M.; O’Neill, J.S.; Wong, G.K.; Chesham, J.; Odell, M.; Lilley, K.S.; et al. Circadian orchestration of the hepatic proteome. Curr. Biol. 2006, 16, 1107-1115.

98. Jouffe, C.; Cretenet, G.; Symul, L.; Martin, E.; Atger, F.; Naef, F.; Gachon, F. The circadian clock coordinates ribosome biogenesis. PLoS Biol. 2013, 11, e1001455. 
99. Newby, L.M.; Jackson, F.R. Regulation of a specific circadian clock output pathway by lark, a putative RNA-binding protein with repressor activity. J. Neurobiol. 1996, 31, 117-128.

100. Sofola, O.; Sundram, V.; Ng, F.; Kleyner, Y.; Morales, J.; Botas, J.; Jackson, F.R.; Nelson, D.L. The Drosophila FMRP and LARK RNA-binding proteins function together to regulate eye development and circadian behavior. J. Neurosci. 2008, 28, 10200-10205.

101. Kojima, S.; Matsumoto,K.; Hirose, M.; Shimada, M.; Nagano, M.; Shigeyoshi, Y.; Hoshino, S.; Ui-Tei, K.; Saigo, K.; Green, C.B.; et al. LARK activates posttranscriptional expression of an essential mammalian clock protein, PERIOD1. Proc. Natl. Acad. Sci. USA 2007, 104, 1859-1864.

102. Kim, D.Y.; Woo, K.C.; Lee, K.H.; Kim, T.D.; Kim, K.T. hnRNP Q and PTB modulate the circadian oscillation of mouse Rev-erb alpha via IRES-mediated translation. Nucleic Acids Res. 2010, 38, 7068-7078.

103. Kim, T.D.; Woo, K.C.; Cho, S.; Ha, D.C.; Jang, S.K.; Kim, K.T. Rhythmic control of AANAT translation by hnRNP Q in circadian melatonin production. Genes Dev. 2007, 21, 797-810.

104. Lee, K.H.; Woo, K.C.; Kim, D.Y.; Kim, T.D.; Shin, J.; Park, S.M.; Jang, S.K.; Kim, K.T. Rhythmic Interaction between Period1 mRNA and hnRNP Q Leads to Circadian Time-Dependent Translation. Mol. Cell. Biol. 2012, 32, 717-728.

105. Morse, D.; Milos, P.M.; Roux, E.; Hastings, J.W. Circadian regulation of bioluminescence in Gonyaulax involves translational control. Proc. Natl. Acad. Sci. USA 1989, 86, 172-176.

106. Mittag, M.; Lee, D.H.; Hastings, J.W. Circadian expression of the luciferin-binding protein correlates with the binding of a protein to the $3^{\prime}$ untranslated region of its mRNA. Proc. Natl. Acad. Sci. USA 1994, 91, 5257-5261.

107. Mittag, M.; Waltenberger, H. In vitro mutagenesis of binding site elements for the clock-controlled proteins CCTR and Chlamy 1. Biol. Chem. 1997, 378, 1167-1170.

108. Kimchi-Sarfaty, C.; Oh, J.M.; Kim, I.W.; Sauna, Z.E.; Calcagno, A.M.; Ambudkar, S.V.; Gottesman, M.M. A "silent" polymorphism in the MDR1 gene changes substrate specificity. Science 2007, 315, 525-528.

109. Zhou, M.; Guo, J.; Cha, J.; Chase, M.; Chen, S.; Barral, J.M.; Sachs, M.S.; Liu, Y. Non-optimal codon usage determines the expression, structure and function of a circadian clock protein. Nature 2013, 495, 111-115.

110. Xu, Y.; Ma, P.; Shah, P.; Rokas, A.; Liu, Y.; Johnson, C.H. Non-optimal codon usage is a mechanism to achieve circadian clock conditionality. Nature 2013, 495, 116-120.

111. Carpen, J.D.; von Schantz, M.; Smits, M.; Skene, D.J.; Archer, S.N. A silent polymorphism in the PER1 gene associates with extreme diurnal preference in humans. J. Hum. Genet. 2006, 51, 1122-1125.

112. Parker, R.; Song, H. The enzymes and control of eukaryotic mRNA turnover. Nat. Struct. Mol. Biol. 2004, 11, 121-127.

113. Dziembowski, A.; Lorentzen, E.; Conti, E.; Seraphin, B. A single subunit, Dis3, is essentially responsible for yeast exosome core activity. Nat. Struct. Mol. Biol. 2007, 14, 15-22.

114. Hartung, S.; Hopfner, K.P. Lessons from structural and biochemical studies on the archaeal exosome. Biochem. Soc. Trans. 2009, 37, 83-87. 
115. Chen, C.Y.; Shyu, A.B. AU-rich elements: Characterization and importance in mRNA degradation. Trends Biochem. Sci. 1995, 20, 465-470.

116. Benjamin, D.; Schmidlin, M.; Min, L.; Gross, B.; Moroni, C. BRF1 protein turnover and mRNA decay activity are regulated by protein kinase B at the same phosphorylation sites. Mol. Cell. Biol. 2006, 26, 9497-9507.

117. Kim, T.D.; Kim, J.S.; Kim, J.H.; Myung, J.; Chae, H.D.; Woo, K.C.; Jang, S.K.; Koh, D.S.; Kim, K.T. Rhythmic Serotonin $N$-Acetyltransferase mRNA degradation is essential for the maintenance of its circadian oscillation. Mol. Cell. Biol. 2005, 25, 3232-3246.

118. Kim, D.Y.; Kwak, E.; Kim, S.H.; Lee, K.H.; Woo, K.C.; Kim, K.T. hnRNP Q mediates a phase-dependent translation-coupled mRNA decay of mouse Period3. Nucleic Acids Res. 2011, 39, 8901-8914.

119. Bartel, D.P. MicroRNAs: Genomics, biogenesis, mechanism, and function. Cell 2004, 116, 281-297.

120. Hammond, S.M. Dicing and slicing: The core machinery of the RNA interference pathway. FEBS Lett. 2005, 579, 5822-5829.

121. Jaskiewicz, L.; Filipowicz, W. Role of Dicer in posttranscriptional RNA silencing. Curr. Top. Microbiol. Immunol. 2008, 320, 77-97.

122. Cheng, H.Y.; Papp, J.W.; Varlamova, O.; Dziema, H.; Russell, B.; Curfman, J.P.; Nakazawa, T.; Shimizu, K.; Okamura, H; Impey, S.; et al. microRNA modulation of circadian-clock period and entrainment. Neuron 2007, 54, 813-829.

123. Gatfield, D.; le Martelot, G.; Vejnar, C.E.; Gerlach, D.; Schaad, O.; Fleury-Olela, F.; Ruskeepaa, A.L.; Oresic, M.; Esau, C.C.; Zdobnov, E.M.; et al. Integration of microRNA miR-122 in hepatic circadian gene expression. Genes Dev. 2009, 23, 1313-1326.

124. Kadener, S.; Menet, J.S.; Sugino, K.; Horwich, M.D.; Weissbein, U.; Nawathean, P.; Vagin, V.V.; Zamore, P.D.; Nelson, S.B.; Rosbash, M. A role for microRNAs in the Drosophila circadian clock. Genes Dev. 2009, 23, 2179-2191.

125. Pegoraro, M.; Tauber, E. The role of microRNAs (miRNA) in circadian rhythmicity. J. Genet. 2008, 87, 505-511.

126. Saus, E.; Soria, V.; Escaramis, G.; Vivarelli, F.; Crespo, J.M.; Kagerbauer, B.; Menchon, J.M.; Urretavizcaya, M.; Gratacos, M.; Estivill, X. Genetic variants and abnormal processing of pre-miR-182, a circadian clock modulator, in major depression patients with late insomnia. Hum. Mol. Genet. 2010, 19, 4017-4025.

127. Xu, S.; Witmer, P.D.; Lumayag, S.; Kovacs, B.; Valle, D. MicroRNA (miRNA) transcriptome of mouse retina and identification of a sensory organ-specific miRNA cluster. J. Biol. Chem. 2007, 282, 25053-25066.

128. Zhou, W.; Li, Y.; Wang, X.; Wu, L.; Wang, Y. MiR-206-mediated dynamic mechanism of the mammalian circadian clock. BMC Syst. Biol. 2011, 5, 141.

(C) 2013 by the authors; licensee MDPI, Basel, Switzerland. This article is an open access article distributed under the terms and conditions of the Creative Commons Attribution license (http://creativecommons.org/licenses/by/3.0/). 